\title{
Effect of alkylglycerone phosphate synthase on the expression profile of circRNAs in the human thyroid cancer cell line FRO
}

\author{
SHASHA HOU ${ }^{1,2^{*}}$, JIAN TAN $^{1 *}$, BING YANG $^{3^{*}}, \mathrm{LU} \mathrm{HE}^{4}$ and $\mathrm{YU} \mathrm{ZHU}^{5}$ \\ ${ }^{1}$ Department of Nuclear Medicine, Tianjin Medical University General Hospital, Tianjin 300052; \\ ${ }^{2}$ Graduate School; Departments of ${ }^{3}$ Cell Biology, and ${ }^{4}$ Anatomy and Histology, School of Basic Medical Sciences, \\ Tianjin Medical University, Tianjin 300070; ${ }^{5}$ Department of Clinical Laboratory, Tianjin Key Laboratory of \\ Cerebral Vessels and Neurodegenerative Diseases, Tianjin Huanhu Hospital, Tianjin 300350, P.R. China
}

Received September 24, 2017; Accepted February 27, 2018

DOI: $10.3892 / 01.2018 .8356$

\begin{abstract}
Thyroid cancer is a common primary tumor in China. Therefore, it is important to investigate the underlying molecular mechanism of thyroid cancer in order to achieve effective individualized treatments. In our previous study, a positive correlation between the expression of alkylglycerone phosphate synthase (AGPS) and the malignant phenotype of thyroid cancer cell lines was identified. The inactivation of AGPS was able to decrease the malignancy of cancer, and inhibit tumor growth and invasion. However, the function of AGPS on thyroid cancer was unclear. In the present study, it was revealed that AGPS was able to regulate the expression of circular RNAs (circRNAs), which may be the mechanism of its anticancer activity. Therefore, the effects of AGPS silencing and knockout on circRNA expression in the thyroid cancer cell line FRO were investigated using circRNAs microarray, and Gene Ontology and Kyoto Encyclopedia of Genes and Genomes pathway enrichment analyses were performed in order to investigate the underlying molecular mechanism of AGPS for the regulation of thyroid cancer through circRNAs.
\end{abstract}

Correspondence to: Professor Yu Zhu, Department of Clinical Laboratory, Tianjin Key Laboratory of Cerebral Vessels and Neurodegenerative Diseases, Tianjin Huanhu Hospital, 6 Jizhao Road, Tianjin 300350, P.R. China

E-mail: zhuyutj@126.com

Professor Lu He, Department of Anatomy and Histology, School of Basic Medical Sciences, Tianjin Medical University, 22 Qixiangtai Road, Tianjin 300070, P.R. China

E-mail: helu@tmu.edu.cn

*Contributed equally

Key words: circular RNAs, thyroid cancer, alkylglycerone phosphate synthase

\section{Introduction}

Thyroid cancer is type of malignant tumor with an increasing incidence in China $(1,2)$. However, the current clinical treatment of thyroid cancer, which involves surgery and/or radiotherapy or chemotherapy, results in low recovery rates and high recurrence rates, indicating that the treatment in not sufficiently effective (3). Therefore, the aim of the present study was to identify a novel target for thyroid cancer and investigate its underlying molecular mechanism.

Alkylglycerone phosphate synthase (AGPS) inactivation is able to decrease the expression of various types of lipid that are crucial for the growth and diffusion of tumor cells, including ether lipids, prostaglandins and acyl phosphatides, thus decreasing cancer pathogenicity $(4,5)$. However, AGPS overexpression is able to increase the survival and migratory ability of tumor cells, including SKOV3 ovarian cancer cells, 231MFP breast cancer cells, C8161 melanoma cells, PC3 prostate cancer cells and primary breast cancer cells, thus promoting tumor growth and invasion (6). In our previous study, a differential expression of AGPS in various thyroid cancer cell lines was observed, which was positively correlated with their malignancy. Therefore, it was hypothesized that AGPS may be a potential target for the diagnosis and treatment of thyroid cancer.

Circular RNAs (circRNAs) are important molecular mediators of cellular genetic changes, which are directly or indirectly associated with the occurrence and development of numerous tumors, including thyroid cancer. circRNAs are abnormally expressed in tumors, and are a newly identified type of non-coding RNA that differ from traditional non-coding linear RNAs by their unique mechanism, including i) as the small RNA [microRNA (miRNA)] sponge regulating the expression of target genes; ii) regulating the activity of RNA polymerase II transcripts; iii) regulating the RNA-binding protein; and iv) combining with ribosomes to participate in protein translation $(7,8)$. Therefore, it was hypothesized that circRNAs may also be potential targets for the diagnosis and treatment of thyroid cancer.

The aim of the present study was to identify an association between AGPS and circRNAs in thyroid cancer. For that purpose, AGPS was silenced and knocked out in FRO cells 
to explore the effects of AGPS on the regulation of circRNA expression in thyroid cancer cells. In addition, Gene Ontology (GO) and Kyoto Encyclopedia of Genes and Genomes (KEGG) pathway enrichment analyses were used to investigate the underlying molecular mechanism of AGPS-regulated biological behavior of thyroid cancer.

\section{Materials and methods}

Cell lines and cell culture. The human thyroid cancer cell lines TPC-1, FTC-133 and FRO were obtained from the American Type Culture Collection (Manassas, VA, USA) and maintained at $37^{\circ} \mathrm{C}$ in a humidified atmosphere with $5 \% \mathrm{CO}_{2}$ in RPMI-1640 medium (Corning Incorporated, Corning, NY, USA) with $10 \%$ fetal bovine serum (Corning Incorporated).

FRO cells were cultured in a 6-well plate for $24 \mathrm{~h}$, and then transfected with an AGPS short hairpin (sh)RNA plasmid (sc-94310-SH; Santa Cruz Biotechnology, Inc., Dallas, TX, USA) (AGPS sh group) or with an AGPS clustered regularly interspaced short palindromic repeats-associated protein-9 nuclease knockout (KO) plasmid (sc-404604; Santa Cruz Biotechnology, Inc.) (AGPS KO group), according to the manufacturer's protocol. Next, fresh culture medium was added, and cells were harvested $72 \mathrm{~h}$ later for experiments.

RNA sample quality control. The total RNA of AGPS SH and AGPS KO FRO cells were extracted using TRIzol ${ }^{\circledR}$ reagent (Invitrogen; Thermo Fisher Scientific, Inc., Waltham, MA, USA). The concentration of the RNA samples was determined by measuring the optical density at $260 \mathrm{~nm}$ using a NanoDrop ND-1000 instrument (NanoDrop; Thermo Fisher Scientific, Inc.). The RNA integrity was assessed by electrophoresis on a $1.5 \%$ denaturing agarose gel. Visualization was performed using SYBR ${ }^{\circledast}$ Green I nucleic acid gel stain (Sigma-Aldricj; Merck KGaA, Darmstadt, Germany) and a Safe Imager ${ }^{\mathrm{TM}} 2.0$ Blue Light Transilluminator (Thermo Fisher Scientific Inc., Waltham, MA, USA).

Labeling and hybridization. Sample labeling and array hybridization were performed according to the manufacturer's protocol (Arraystar Super RNA Labeling Kit; Arraystar, Inc., Rockville, MD, USA). Briefly, total RNAs from AGPS SH and AGPS KO FRO cells were digested with RNase R (Epicentre; Illumina, Inc., San Diego, CA, USA) to remove linear RNAs and enrich the sample in circRNAs. Then, the enriched circRNAs were amplified and transcribed into fluorescent complementary RNA (cRNA) utilizing a random priming method (Arraystar Super RNA Labeling kit; Arraystar, Inc.). The labeled cRNAs were purified using an RNeasy Mini kit (Qiagen, Inc., Valencia, CA, USA). The concentration and specific activity of the labeled cRNAs (pmol cyanin $3 / \mu \mathrm{g}$ cRNA) were determined using a NanoDrop ND-1000 instrument. Next, $1 \mu \mathrm{g}$ each labeled cRNA was fragmented by adding $5 \mu 1$ 10X Blocking Agent (Arraystar Super RNA Labeling Kit) and $1 \mu \mathrm{l} 25 \mathrm{X}$ Fragmentation Buffer (Arraystar Super RNA Labeling Kit), and the mixture was heated at $60^{\circ} \mathrm{C}$ for 30 min before adding $25 \mu \mathrm{l} 2 \mathrm{X}$ Hybridization Buffer (Arraystar Super RNA Labeling Kit) to dilute the labeled
cRNAs. Subsequently, $50 \mu 1$ Hybridization Solution (Arraystar Super RNA Labeling Kit) was dispensed into the gasket slide and assembled to create the circRNA expression microarray slide. The slides were incubated at $65^{\circ} \mathrm{C}$ for $17 \mathrm{~h}$ in an Agilent Microarray Hybridization Oven (Agilent Technologies, Inc., Santa Clara, CA, USA). The hybridized arrays were washed, fixed and scanned using an Agilent Microarray Scanner G2505C (Agilent Technologies, Inc.).

Reverse transcription-quantitative polymerase chain reaction $(R T-q P C R)$ assay. RNA was isolated using QIAzol (Qiagen, Inc.) according to the manufacturer's protocol. SYBR Green I dye (Takara Biotechnology Co., Ltd., Dalian, China) was used for RT in an Applied Biosystems ${ }^{\circledR} 7500$ Real-Time PCR system (Applied Biosystems; Thermo Fisher Scientific, Inc.), and the concentration of circRNAs in the aforementioned total RNA was quantified. The sequences of the primers used for RT-qPCR are listed in Table I. The thermocycling conditions used were as follows: Denaturation at $95^{\circ} \mathrm{C}$ for $10 \mathrm{~min}$, followed by 40 cycles at $95^{\circ} \mathrm{C}$ for $15 \mathrm{sec}$ and $60^{\circ} \mathrm{C}$ for $60 \mathrm{sec}$, and a final elongation at $95^{\circ} \mathrm{C}$ for $15 \mathrm{sec}, 60^{\circ} \mathrm{C}$ for $60 \mathrm{sec}$ and $95^{\circ} \mathrm{C}$ for $15 \mathrm{sec}$. $\beta$-actin was used as the internal reference, and the $2^{-\triangle \triangle C q}$ method (9) was used for calculating circRNA expression ratios.

\section{Data analysis}

Raw data collection. Scanned images were imported into Agilent Feature Extraction Software 12.0 (Agilent Technologies, Inc.) for raw data extraction.

Expression profiling data. Quantile normalization of raw data and subsequent data processing were performed using the R software limma package (10). Next, volcano plot (statistics) was performed (11).

Differentially expressed data. When comparing two groups of profile differences (such as disease vs. control), the fold change (FC), which was defined as the mean of the ratio of the group, between the groups for each circRNA was computed. The statistical significance of the difference was estimated using Student's t-test. circRNAs exhibiting $\mathrm{FC}=2$ and $\mathrm{P}=0.05$ were selected as significantly differentially expressed. The analysis outputs were filtered, and the differentially expressed circRNAs were ranked according to their FC and P-value, using the Sort \& Filter Data functionality of Microsoft Excel 2007 (Microsoft Corporation, Redmond, WA, USA). The significantly differentially expressed circRNAs $(F C \geq 2.0)$ was considered to indicate a significantly differentially expressed circRNAs

Statistical analysis. A heatmap was constructed using HemI 1.0 (12). Cluster analysis was performed by complete linkage (13). GO and KEGG analyses were performed using DAVID 6.8 (14). $-\log 10$ (P-value) $>2$ was considered to indicate a significant threshold in GO analysis. Data analysis was conducted using SPSS software (version 11.0; SPSS, Inc., Chicago, IL, USA). One-way analysis of variance and Tukey's test were applied for intergroup analysis. $\mathrm{P}<0.05$ was considered to indicate a statistically significant difference. 
Table I. Primer sequences.

\begin{tabular}{|c|c|}
\hline Gene & Primer sequence $\left(5^{\prime}-3^{\prime}\right)$ \\
\hline \multirow[t]{2}{*}{ AGPS } & Forward: ACCAGATTCCCTGGAGTTCA \\
\hline & Reverse: GAACCACCAGGTCCTCGATA \\
\hline \multirow[t]{2}{*}{ hsa_circRNA_406841 } & Forward: ATGGAGCACCCTGGGAAAA \\
\hline & Reverse: TTTGCGACTCACTCTTCTGG \\
\hline \multirow[t]{2}{*}{ hsa_circRNA_000905 } & Forward: GCCAAGAAGCCACTGACTC \\
\hline & Reverse: CCGTACCCACAAAGCAGTC \\
\hline \multirow[t]{2}{*}{ hsa_circRNA_019252 } & Forward: CTACAAGCCCGCGC \\
\hline & Reverse: CTATACATTTATTGAGTAAAAACAAAAC \\
\hline \multirow[t]{2}{*}{ hsa_circRNA_089761 } & Forward: ATTTTGTTTTCAATTAGGGAGAT \\
\hline & Reverse: GCACATGCAGCGCAAGTA \\
\hline \multirow[t]{2}{*}{ hsa_circRNA_006050 } & Forward: GATCATTCAGGTTCTTCCAGG \\
\hline & Reverse: TTCTCTTGGAAACGTTCAGAAG \\
\hline \multirow[t]{2}{*}{ hsa_circRNA_074298 } & Forward: TTTGGCAATGAATAAACTGACC \\
\hline & Reverse: CCGAGCAAAAGGAACTCCG \\
\hline \multirow[t]{2}{*}{ hsa_circRNA_066556 } & Forward: AGTGGCATGATCGCGTCT \\
\hline & Reverse: ATTTCGACTCACTGCTTCACCA \\
\hline \multirow[t]{2}{*}{ hsa_circRNA_101321 } & Forward: TTTGGTGCATATTTGGGTCT \\
\hline & Reverse: CTGTAACATGGCCTTGAGGA \\
\hline \multirow[t]{2}{*}{ hsa_circRNA_023016 } & Forward: GGGTAGTGGGATGTGTGTCC \\
\hline & Reverse: AAACCATTTATTTCACCGGAA \\
\hline \multirow[t]{2}{*}{ hsa_circRNA_019744 } & Forward: ACACGTCAGCTCCCTCGCCGCCCTG \\
\hline & Reverse: ATCGGAACGACTTTATTTCAGTA \\
\hline \multirow[t]{2}{*}{ hsa_circRNA_404686 } & Forward: GACCAGGTGATTTTCAAAGC \\
\hline & Reverse: ATAATCAAAGGAATGGACGC \\
\hline \multirow[t]{2}{*}{ hsa_circRNA_000367 } & Forward: GTCCCAGTAAGCACTCTGTTC \\
\hline & Reverse: AATCGTATGGAATGGACCTG \\
\hline \multirow{2}{*}{ hsa_circRNA_001729 } & Forward: AGGAGCCCAGACACAGCAG \\
\hline & Reverse: CCATTACACTGTAGCCAGAATG \\
\hline \multirow[t]{2}{*}{ hsa_circRNA_004183 } & Forward: ATAGCTCGCAGTCGGCC \\
\hline & Reverse: CCTGAGAACCTCGTGGAAT \\
\hline \multirow[t]{2}{*}{ hsa_circRNA_100790 } & Forward: AAAACGGTTCCTTTGGTATC \\
\hline & Reverse: TGGAATGGAGCTGCATTTAC \\
\hline \multirow[t]{2}{*}{ hsa_circRNA_104270 } & Forward: GTGAGAGGTTTGCAAGGATTT \\
\hline & Reverse: GGGAAAGGATCTGGAATGG \\
\hline \multirow[t]{2}{*}{ hsa_circRNA_102049 } & Forward: TACTTCAGATTTTCCTGTCCT \\
\hline & Reverse: AATGGCTGTGTCAGCAGTTTT \\
\hline \multirow[t]{2}{*}{ hsa_circRNA_406494 } & Forward: AGGAACTATAGATTTAGCTTATTGT \\
\hline & Reverse: ATGAAACAATTTGCTTGGCT \\
\hline \multirow[t]{2}{*}{ hsa_circRNA_100787 } & Forward: CATGCAAACGGTGGTAAATT \\
\hline & Reverse: CTTTTACATTGATTCCACTGCT \\
\hline \multirow[t]{2}{*}{ hsa_circRNA_082319 } & Forward: AAGCAACGATGTGCTGAGCT \\
\hline & Reverse: GTTTCAGCTGGCTGGCTAGA \\
\hline \multirow[t]{2}{*}{$\beta$-actin } & Forward: AGGCACCAGGGCGTGAT \\
\hline & Reverse: GCCCACATAGGAATCCTTCTGAC \\
\hline
\end{tabular}

AGPS, alkylglycerone phosphate synthase; circRNA, circular RNA; hsa, Homo sapiens.

\section{Results}

Expression of AGPS in thyroid cancer cell lines. The expression of AGPS was analyzed in different thyroid cancer cells, and low levels of AGPS were detected in TPC-1 and FTC-133 cells, whereas highest level of AGPS were observed in tumors of the FRO cells $(\mathrm{P}<0.05)$, as depicted in Fig. 1. This result indicated that there was an expression of AGPS in thyroid cancer. Therefore, the effect of AGPS on the circRNAs in thyroid cancer was explored using FRO cells AGPS was investigated in tumor cells to analyze the mechanism, therefore TPC-1 cells were selected as control.

Cluster analysis of differentially expressed circRNAs. Cluster analysis can be used to analyze genetic distance 


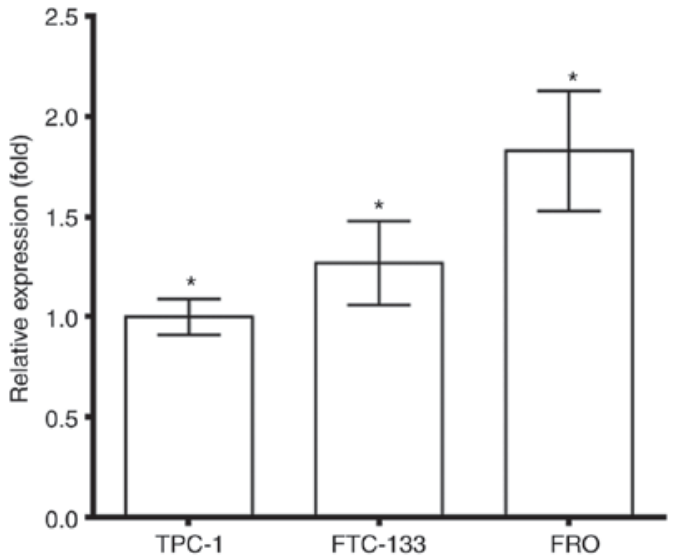

Figure 1. Expression of AGPS in thyroid cancer cell lines. AGPS expression in the thyroid cancer cell lines TPC-1, FTC-133 and FRO was evaluated using reverse transcription-quantitative polymerase chain reaction analysis. ${ }^{*} \mathrm{P}<0.05$ vs. TPC-1. AGPS, alkylglycerone phosphate synthase.

or similarity and gene expression patterns (15). In the present study, the control, AGPS sh and AGPS KO groups were selected for cluster analysis of circRNAs, and the three samples were compared to identify the differences in circRNA expression. The green represents a decreased expression of circRNAs and the red represents an increased expression of circRNAs in Fig. 2. Fig. 2 indicates that the expression patterns of the control, AGPS sh and AGPS KO groups are different, and therefore, the AGPS sh and AGPS KO may induce the alteration in circRNAs in FRO cells.

Regulation of circRNA expression by AGPS. In the circRNA expression scatter diagrams presented in Fig. 3A, all circRNA expression patterns are compared in pairs between the control, AGPS sh and AGPS KO groups. The difference in circRNA number in the histogram of Fig. 3B indicates that the number of circRNAs which changed expression in the AGPS KO group was greater than the AGPS sh group. The Venn diagram depicted the comparison of changed expression circRNAs in the control, AGPS sh group and AGPS KO group (Fig. 4). The 50 genes with the highest fold-change differently expressed circRNAs are presented in Table II.

To further confirm the regulatory effect of AGPS on circRNA expression in the human thyroid carcinoma cell line FRO, the top 10 circRNAs with increased expression [including Homo sapiens (hsa)_circRNA_406841, hsa_circRNA_000905 and hsa_circRNA_019252] and the top 10 circRNAs with decreased expression (including hsa_circRNA_404686, hsa circRNA_000367 and hsa_circRNA_001729) in the AGPS $\mathrm{KO}$ group compared with the control group were verified using RT-qPCR. The results were consistent with the gene chip results. As presented in Fig. 5, the expression of hsa_circRNA_406841, hsa_circRNA_000905, hsa_circRNA_019252, hsa_circRNA_089761, hsa_circRNA_006050, hsa_circRNA_074298, hsa_circRNA_066556, hsa_circRNA_101321, hsa_circRNA_023016 and hsa_ circRNA_019744 was increased in the AGPS sh and KO groups compared with the control group $(\mathrm{P}<0.05)$. By contrast, the expression of hsa_circRNA_404686, hsa_circRNA_000367,

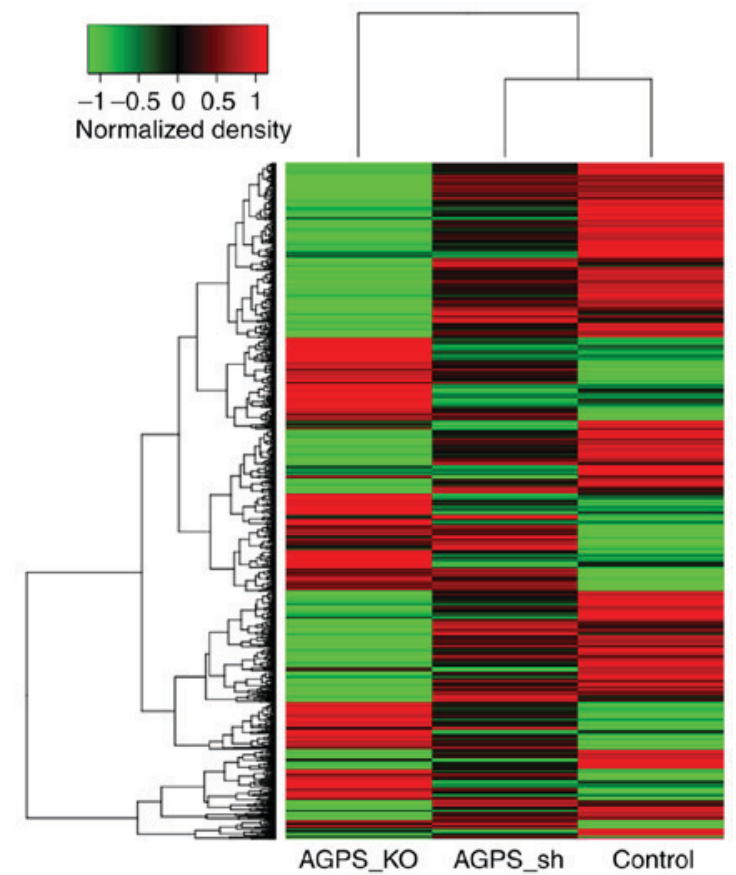

Figure 2. Heatmap of circRNA expression data. The different expressions of circRNAs were depicted in the heatmap. Green represents a decreased expression of circRNAs and red represents an increased expression of circRNAs. Cluster analysis demonstrated that AGPS sh and AGPS KO could induce the alteration in circRNAs expression in FRO cells. circRNA, circular RNA; AGPS, alkylglycerone phosphate synthase; sh, short hairpin; KO, knockout.

hsa_circRNA_001729， hsa_circRNA_004183, hsa_circRNA_100790, hsa_circRNA_104270, hsa_circRNA_102049, hsa_circRNA_406494, hsa_ circRNA_100787 and hsa_circRNA_082319 was decreased in the AGPS sh and KO groups compared with the control group $(\mathrm{P}<0.05)$.

GO enrichment analysis. GO enrichment analysis was used to classify the differently expressed circRNAs in the control, AGPS sh and AGPS KO groups in terms of the life processes regulated by these circRNAs. The results of silencing AGPS were compared with the control and AGPS KO groups in Fig. 6A-D, whereas the AGPS KO group was compared with the control group in terms of molecular function, biological process and cellular component in Fig. 6E and F. The main biological processes were cell adhesion, cell cycle and metabolism; the main cellular components were nucleoplasm, membrane and cytoskeleton; and the main molecular functions were protein binding, poly(A) RNA binding and adenosine 5 '-triphosphate binding.

KEGG pathway enrichment analysis. The KEGG pathway enrichment analysis was similar to that of GO enrichment, which was used to identify genes enriched in a pathway to analyze the effect of AGPS silencing and KO on regulatory functions and signaling pathways. The results of AGPS sh compared with the control and AGPS KO groups are presented in Fig. 7A and B, whereas AGPS KO was compared with the control group in terms of regulatory functions and signaling pathways in Fig. 7C. It was observed that activity 

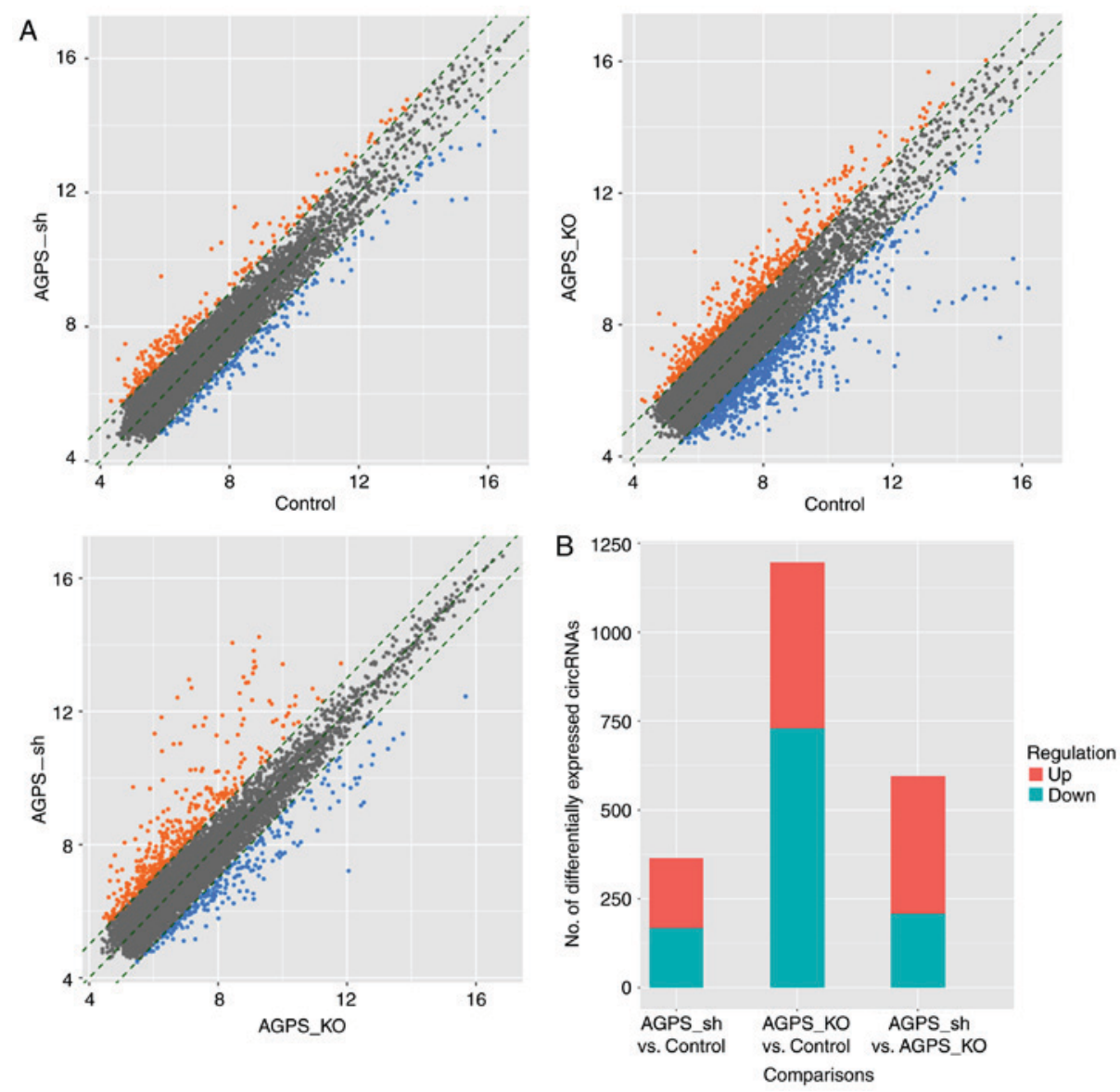

Figure 3. Comparison of differentially expressed circRNAs. (A) Differentially expressed circRNAs among the different groups were identified by scatter plots. The orange points represent $>2$-fold upregulation, whereas the blue points represent $>2$-fold downregulation. (B) Differences in circRNA expression among the three groups. circRNA, circular RNA; AGPS, alkylglycerone phosphate synthase; sh, short hairpin; KO, knockout.

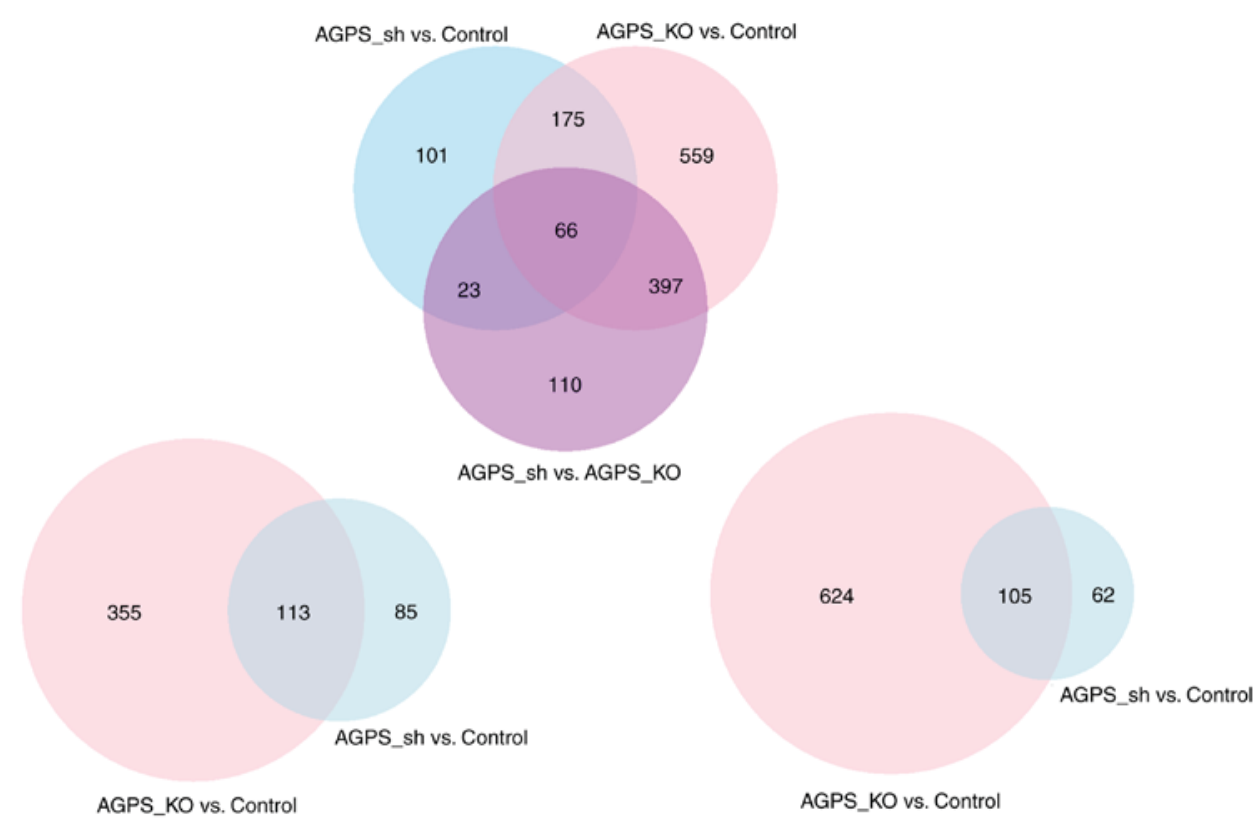

Figure 4. Venn diagrams of differentially expressed circular RNAs in the three groups. The Venn diagrams represent the shared modified genes between groups: AGPS_sh vs. control; AGPS_KO vs. control; and AGPS_sh vs. AGPS_KO, in FRO cell lines. AGPS, alkylglycerone phosphate synthase; sh, short hairpin; KO, knockout.

of the cyclic guanosine 3',5'-monophosphate (cGMP)-protein kinase $\mathrm{G}(\mathrm{PKG})$ and mitogen-activated protein kinase (MAPK) signaling pathways was increased, whereas the regulation of biosynthesis and metabolism was decreased. 


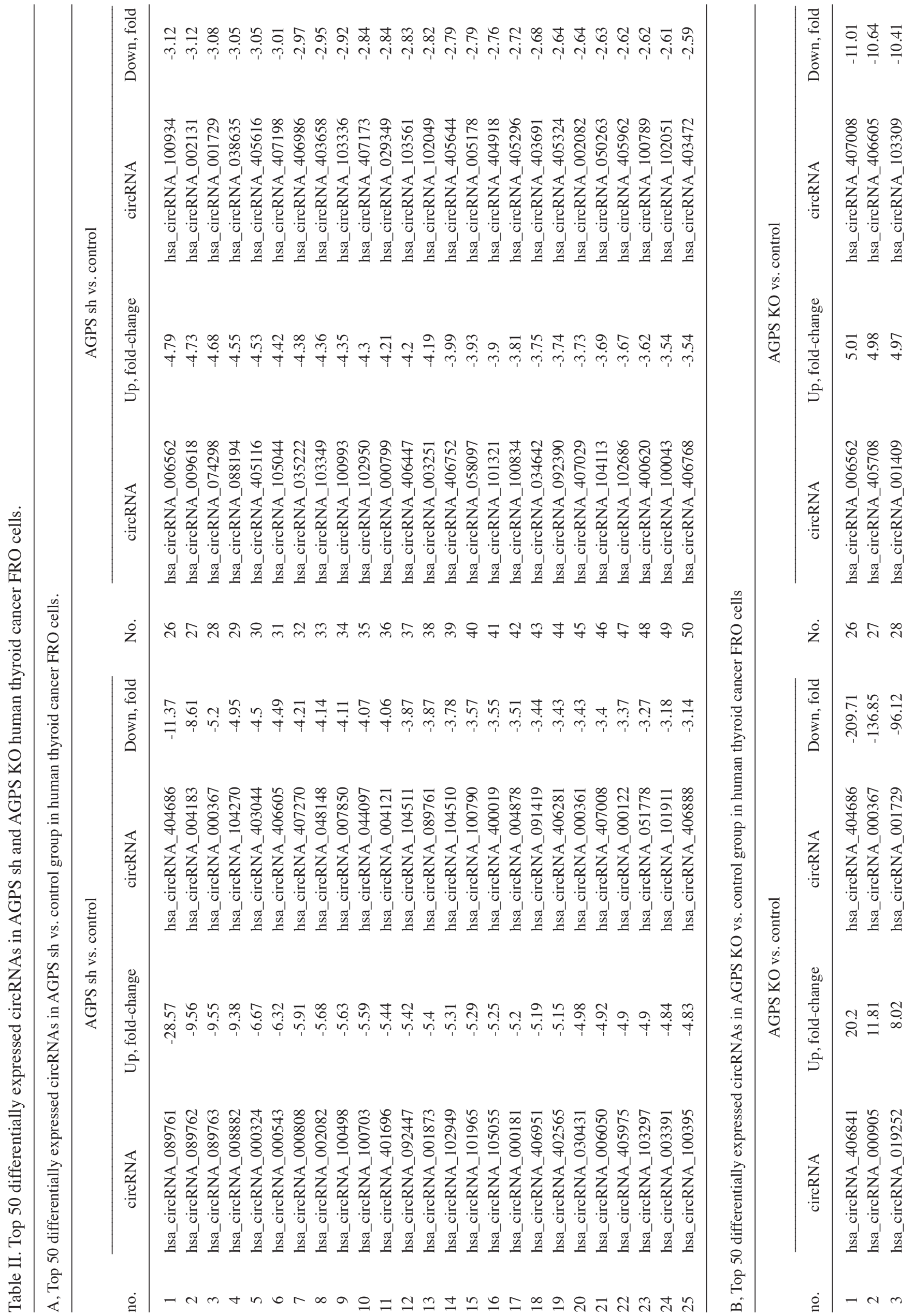




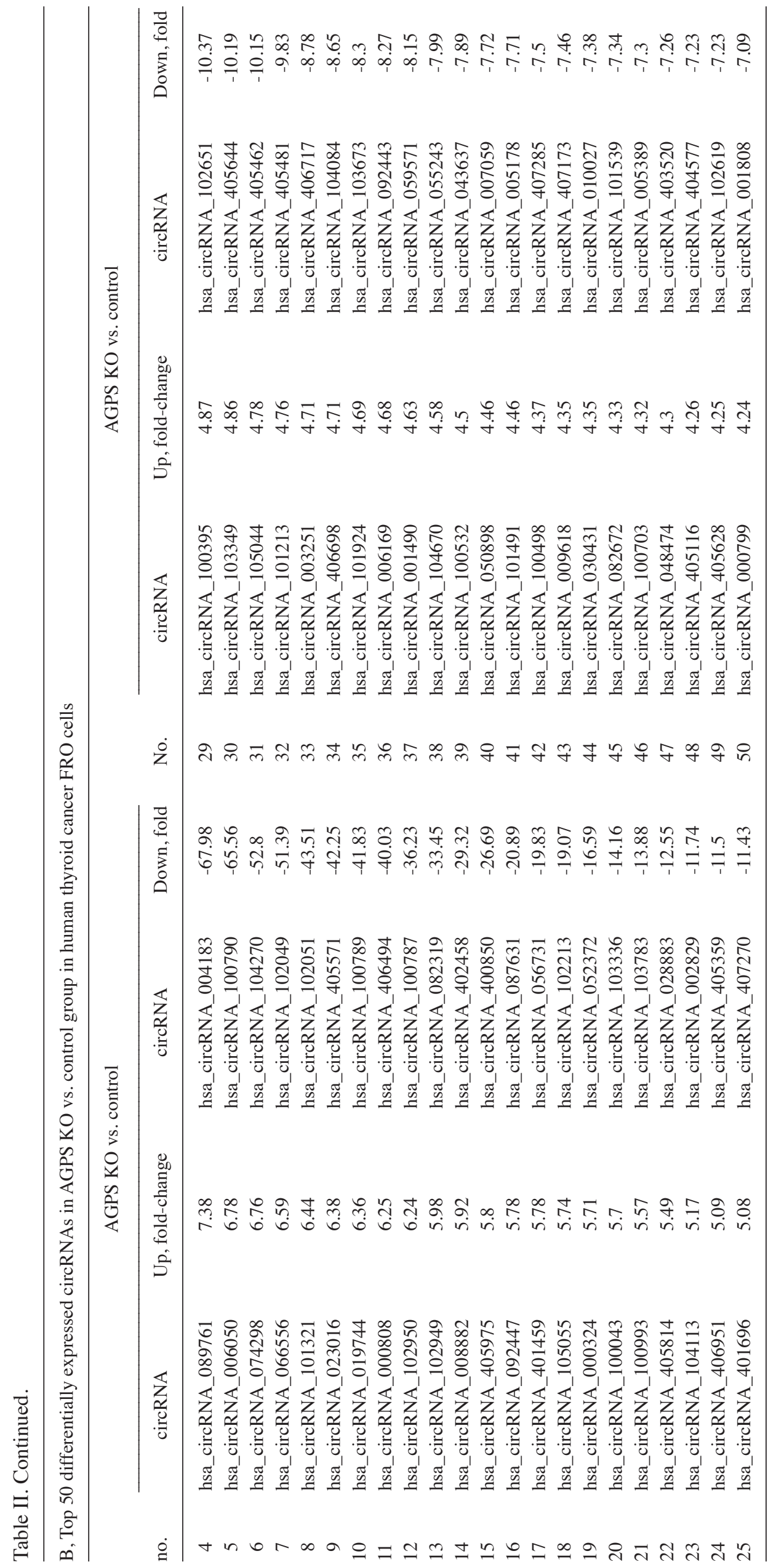




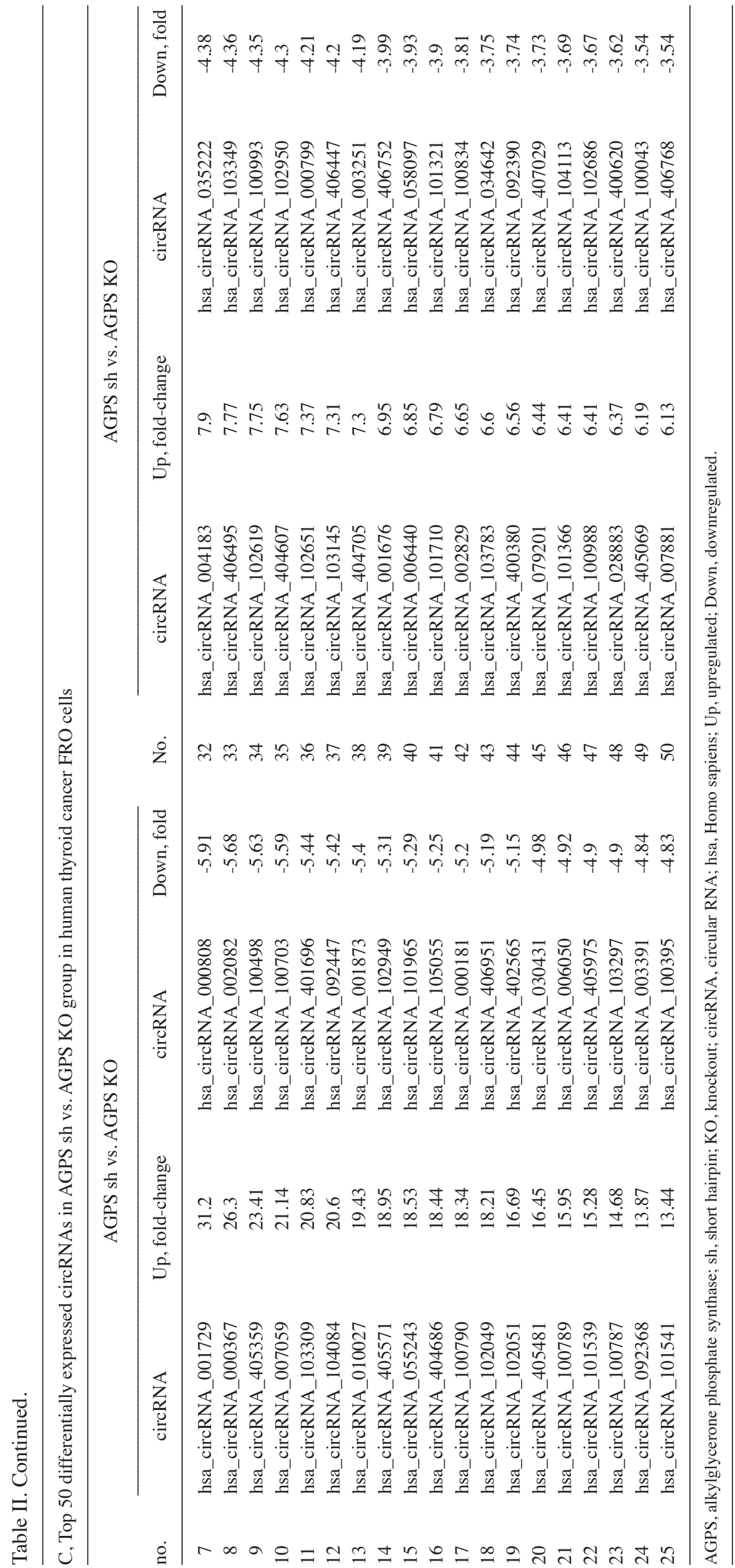




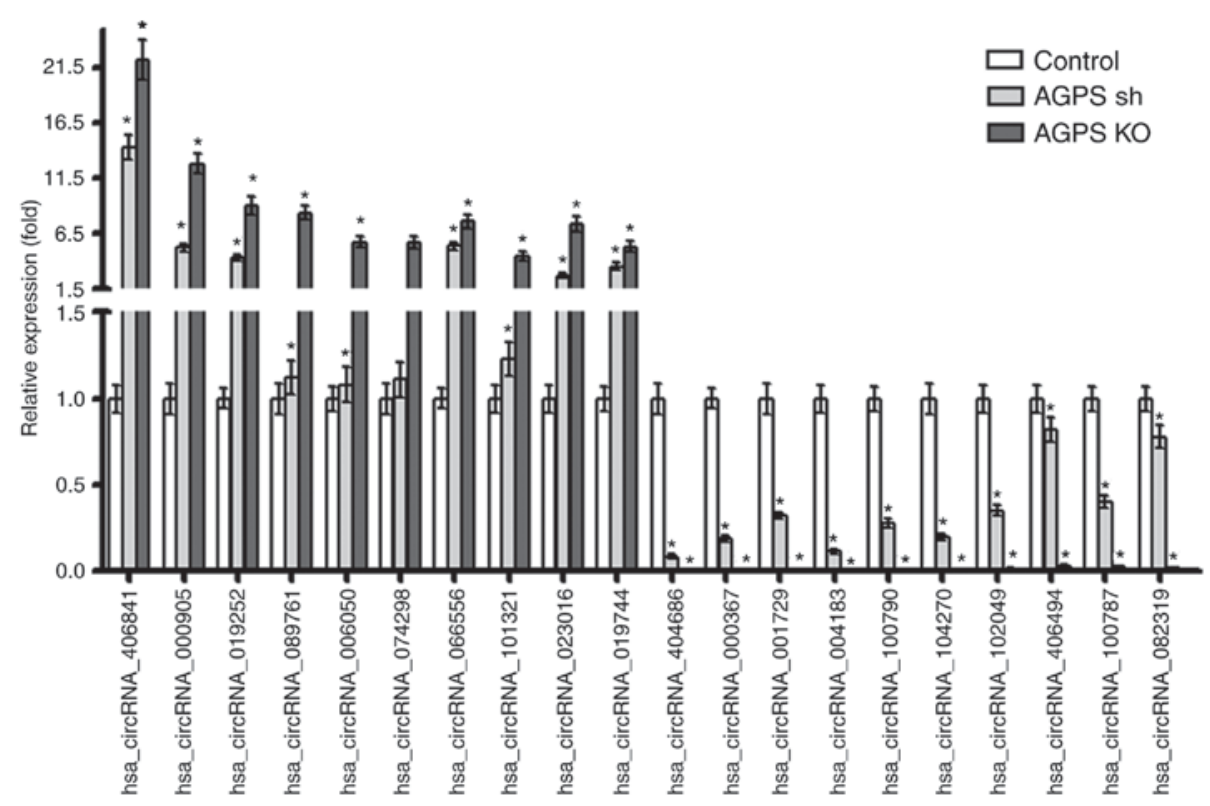

Figure 5. Validation of differentially expressed circRNAs using RT-qPCR. The top 10 circRNAs with increased and decreased expression in the AGPS KO group compared with the control group by RT-qPCR were identified. " $\mathrm{P}<0.05$ vs. control. circRNA, circular RNA; RT-qPCR, reverse transcription-quantitative polymerase chain reaction; AGPS, alkylglycerone phosphate synthase; sh, short hairpin; KO, knockout; hsa, Homo sapiens.
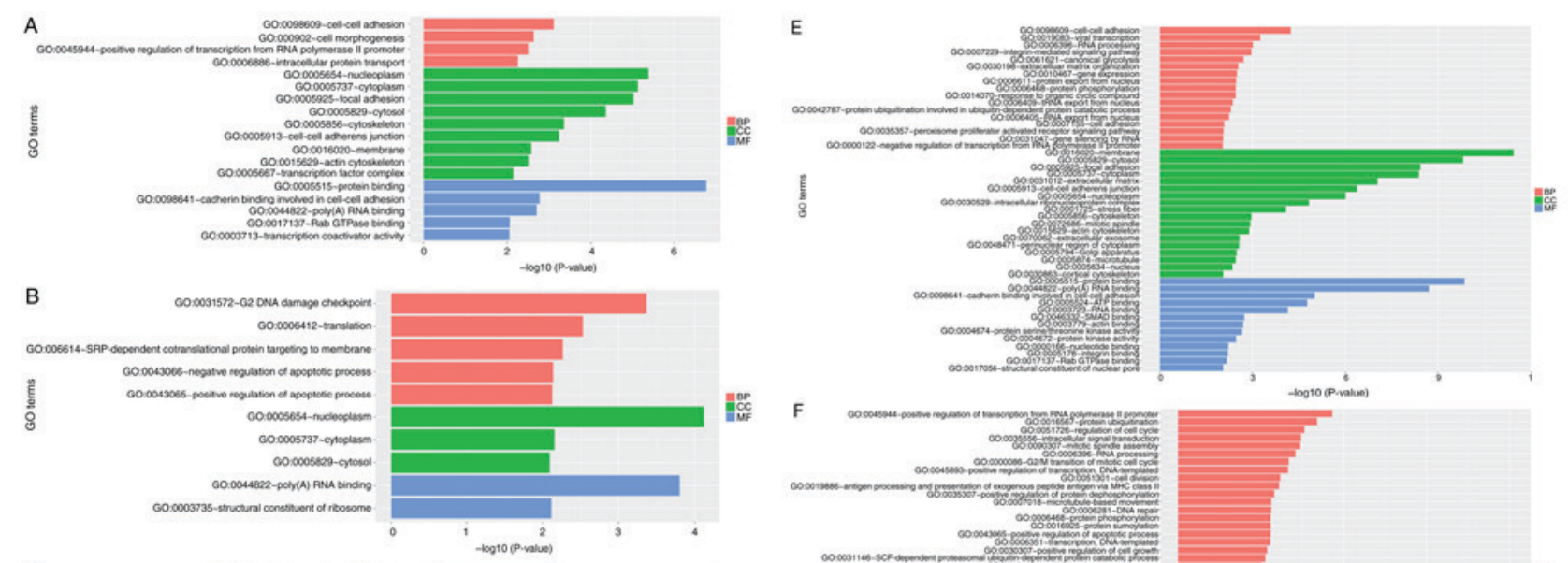

C
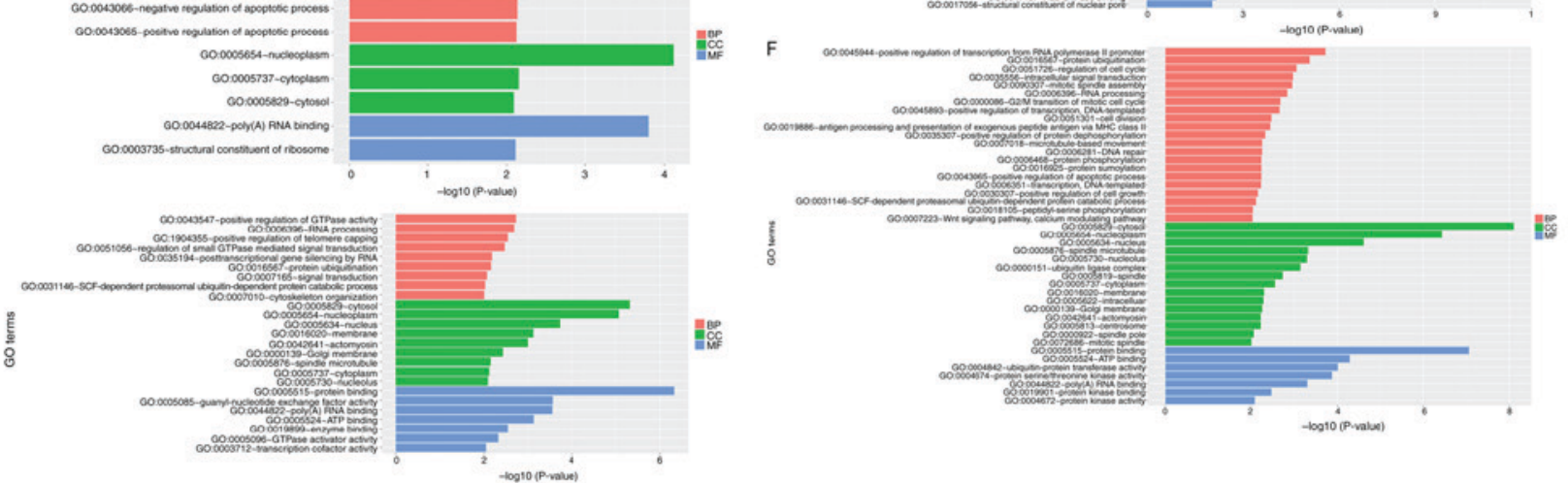

D

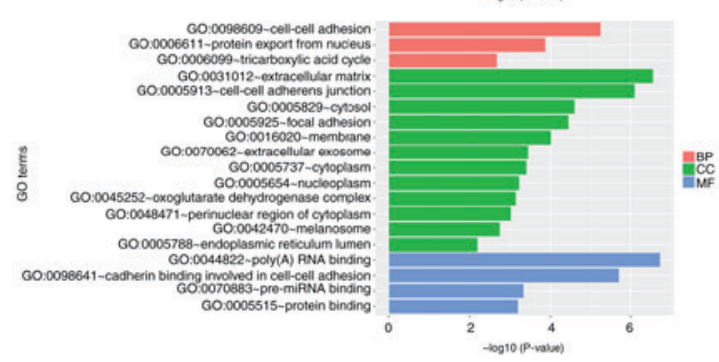

Figure 6. GO analysis of AGPS silencing in thyroid cancer FRO cells. GO analysis was performed on the up- and downregulated genes identified in the AGPS sh, AGPS KO and control thyroid cancer FRO cells. The y-axis represented gene ontology entries, the x-axis represented - $\log 10$ (P-value). - $\log 10$ (P-value) $>2$ was considered to indicate a significant threshold. (A) Upregulation and (B) downregulation of MF, BP and CC by GO analysis of AGPS silencing in FRO cells compared with control thyroid cancer FRO cells. (C) Upregulation and (D) downregulation of MF, BP and CC by GO analysis of AGPS silencing in FRO cells compared with AGPS KO in thyroid cancer FRO cells. (E) Upregulation and (F) downregulation of MF, BP and CC by GO analysis of AGPS KO compared with control thyroid cancer FRO cells. GO, Gene Ontology; AGPS, alkylglycerone phosphate synthase; sh, short hairpin; KO, knockout; MF, molecular function; BP, biological process; CC, cellular component. 

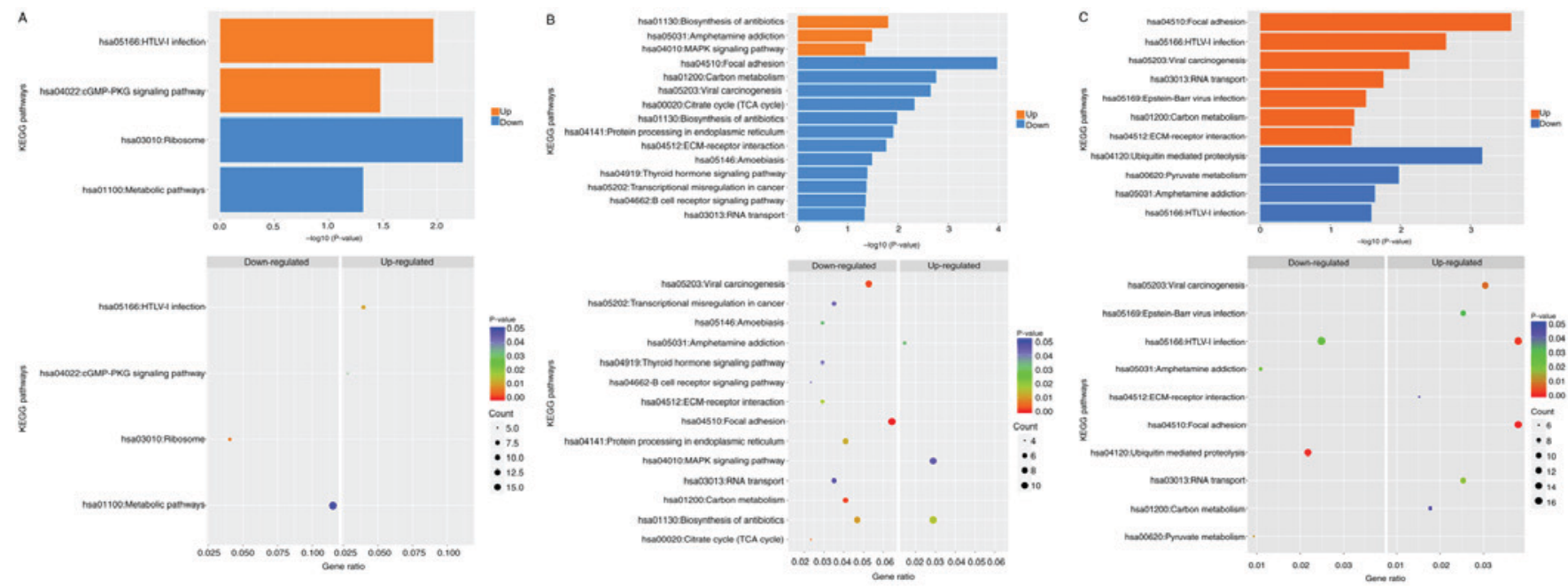

Figure 7. KEGG pathway enrichment analysis of AGPS silencing in thyroid cancer FRO cells. KEGG pathway enrichment analysis is represented by histograms and scatter diagrams. Orange represents the pathway of upregulation gene enrichment, whereas blue represents the downregulated pathway of gene enrichment. The left and right sides of the scatter diagrams correspond to the downregulated and upregulated gene enrichment pathways, respectively. The $\mathrm{x}$-axes represent the proportion of enriched genes, whereas the y-axes contain the name of the KEGG pathway. If up- or downregulated genes were enriched in a certain pathway, this pathway was represented as a point in the graph, the color of which indicated a significant P-value, whereas its size represented the number of differentially expressed genes in that particular pathway. Effect of regulatory function and signaling pathway by KEGG pathway enrichment analysis of AGPS silencing compared with (A) control and (B) KO thyroid cancer FRO cells. (C) Effect of regulatory function and signaling pathway by KEGG pathway enrichment analysis of AGPS KO compared with control thyroid cancer FRO cells. KEGG, Kyoto Encyclopedia of Genes and Genomes; AGPS, alkylglycerone phosphate synthase; KO, knockout; hsa, Homo sapiens.

\section{Discussion}

The occurrence and development of thyroid cancer is a complex biological process (16). The abnormal gene expression involved in signal transduction in tumor cells regulates a series of functional genes abnormal expression, which leads to the acquisition by tumor cells of various characteristics that are different from those of normal cells, and induces cell carcinogenesis, malignant proliferation, invasion and apoptosis resistance (17-20).

AGPS has an increased expression in tumor cells and may regulate cancer progression via lipid metabolism. Our previous study used shRNA technology to knock out AGPS expression in thyroid cancer cells, which significantly decreased the proliferation and invasion of tumor cells in vitro, leading to cell cycle arrest and recovery of sensitivity towards cisplatin by the cisplatin-resistant U87MG/DDP cells, inducing cell apoptosis and inhibition of lipid expression (including lysophosphatidic acid and arachidic acid) in cancer $(4,5)$.

Our study revealed AGPS expression in different malignant thyroid cancer cell lines. Therefore, AGPS was considered as a potential novel target of thyroid cancer. In order to understand the underlying molecular mechanism of thyroid cancer, the malignant thyroid cancer cell line FRO was used in the present study to explore the association between AGPS and circRNAs in thyroid cancer.

The specificity of circRNAs is markedly tissue-specific and developmental stage-dependent (21). These molecules have potential as novel thyroid cancer diagnostic markers and therapeutic targets (16). The present study investigated the effect of silencing or knocking out AGPS expression in thyroid cancer cells, and identified that AGPS silencing or KO was able to regulate the expression of circRNAs. This regulatory function was further confirmed using RT-qPCR.
To the best of our knowledge, no studies on the function and predicted expression of circRNAs in thyroid cancer have been published to date. The present study revealed that various circRNAs were able to regulate the functions of cell adhesion, cell cycle and metabolism in thyroid cancer. It was observed that the downregulation of AGPS and circRNAs expression had a significant effect on the regulation of biological functions, including cell adhesion, cell cycle and metabolism in various tumor types; however, their effects on the thyroid cancer was not explored in the present study. Therefore, the function of AGPS and circRNAs in thyroid cancer will be investigated in future studies as a result of the foundation from the present study.

\section{Acknowledgements}

Not applicable.

\section{Funding}

The present study was supported by the National Natural Science Foundation of China (grant no. 31501159), Tianjin Public Health Key Research Project (grant no. 15KG108), Tianjin Science and Technology Key Project on Chronic Diseases Prevention and Treatment (grant no. 16ZXMJSY00020) and the Special Program of Talents Development for Excellent Youth Scholars in Tianjin, China (grant no. TJTZJH-QNBJRC-2-9).

\section{Availability of data and materials}

All data generated or analyzed during this study are included in this published article. 


\section{Authors' contributions}

YZ was responsible for the conception and design of the study. $\mathrm{SH}$ and BY were responsible for acquisition of data. LH and JT were responsible for data interpretation.

\section{Ethics approval and consent to participate}

Not applicable.

\section{Consent for publication}

Not applicable.

\section{Competing interests}

The authors declare that they have no competing interests.

\section{References}

1. Liu JW, Chen C, Loh EW, Chu CC, Wang MY, Ouyang HJ, Chang YT, Zhuang WZ, Chou CW, Huang DJ, et al: Tyrosine kinase inhibitors for advanced or metastatic thyroid cancer: A meta-analysis of randomized controlled trials. Curr Med Res Opin: 1-9, 2017.

2. Zheng R, Zeng H, Zhang S and Chen W: Estimates of cancer incidence and mortality in China, 2013. Chin J Cancer 36: 66, 2017.

3. Zhu L, Li XJ, Kalimuthu S, Gangadaran P, Lee HW, Oh JM, Baek SH, Jeong SY, Lee SW, Lee J and Ahn BC: Natural killer cell (NK-92MI)-based therapy for pulmonary metastasis of anaplastic thyroid cancer in a nude mouse model. Front Immunol 8: 816, 2017.

4. Zhu Y, Zhu L, Lu L, Zhang L, Zhang G, Wang Q and Yang P: Role and mechanism of the alkylglycerone phosphate synthase in suppressing the invasion potential of human glioma and hepatic carcinoma cells in vitro. Oncol Rep 32: 431-436, 2014.

5. Zhu Y, Liu XJ, Yang P, Zhao M, Lv LX, Zhang GD, Wang Q and Zhang L: Alkylglyceronephosphate synthase (AGPS) alters lipid signaling pathways and supports chemotherapy resistance of glioma and hepatic carcinoma cell lines. Asian Pac J Cancer Prev 15: 3219-3226, 2014

6. Piano V, Benjamin DI, Valente S, Nenci S, Marrocco B, Mai A, Aliverti A, Nomura DK and Mattevi A: Discovery of inhibitors for the ether lipid-generating enzyme AGPS as anti-cancer agents. ACS Chem Biol 10: 2589-2597, 2015.

7. Liang HF, Zhang XZ, Liu BG, Jia GT and Li WL: Circular RNA circ-ABCB10 promotes breast cancer proliferation and progression through sponging miR-1271. Am J Cancer Res 7: 1566-1576, 2017.
8. Huang YS, Jie N, Zou KJ and Weng Y: Expression profile of circular RNAs in human gastric cancer tissues. Mol Med Rep 16: 2469-2476, 2017.

9. Livak KJ and Schmittgen TD: Analysis of relative gene expression data using real-time quantitative PCR and the 2(-Delta Delta C(T)) method. Methods 25: 402-408, 2001.

10. Ritchie ME, Phipson B, Wu D, Hu Y, Law CW, Shi W and Smyth GK: limma powers differential expression analyses for RNA-sequencing and microarray studies. Nucleic Acids Res 43: e47, 2015.

11. Li W: Volcano plots in analyzing differential expressions with mRNA microarrays. J Bioinform Comput Biol 10: 1231003 , 2012.

12. Deng W, Wang Y, Liu Z, Cheng H and Xue Y: HemI: A toolkit for illustrating heatmaps. PLoS One 9: e111988, 2014

13. Bae HW, Rho S, Lee HS, Lee N, Hong S, Seong GJ, Sung KR and Kim CY: Hierarchical cluster analysis of progression patterns in open-angle glaucoma patients with medical treatment. Invest Ophthalmol Vis Sci 55: 3231-3236, 2014.

14. Huang da W, Sherman BT and Lempicki RA: Systematic and integrative analysis of large gene lists using DAVID bioinformatics resources. Nat Protoc 4: 44-57, 2009.

15. Gong P, Madak-Erdogan Z, Li J, Cheng J, Greenlief CM, Helferich W, Katzenellenbogen JA and Katzenellenbogen BS: Transcriptomic analysis identifies gene networks regulated by estrogen receptor $\alpha(E R \alpha)$ and $E R \beta$ that control distinct effects of different botanical estrogens. Nucl Recept Signal 12: e001, 2014.

16. Peng N, Shi L, Zhang Q, Hu Y, Wang N and Ye H: Microarray profiling of circular RNAs in human papillary thyroid carcinoma. PLoS One 12: e0170287, 2017.

17. Zhao L, Sun H, Kong H, Chen Z, Chen B and Zhou M: The Lncrna-TUG1/EZH2 axis promotes pancreatic cancer cell proliferation, migration and EMT phenotype formation through sponging Mir-382. Cell Physiol Biochem 42: 2145-2158, 2017.

18. Li Z, Zhao $\mathrm{K}$ and Tian $\mathrm{H}$ : Integrated analysis of differential expression and alternative splicing of non-small cell lung cancer based on RNA sequencing. Oncol Lett 14: 1519-1525, 2017.

19. Honjo H, Toh Y, Sohda M, Suzuki S, Kaira K, Kanai Y, Nagamori S, Oyama T, Yokobori T, Miyazaki T and Kuwano H: Clinical significance and phenotype of MTA1 expression in esophageal squamous cell carcinoma. Anticancer Res 37: 4147-4155, 2017.

20. Liu $\mathrm{H}$ and Ye $\mathrm{H}$ : Screening of the prognostic targets for breast cancer based co-expression modules analysis. Mol Med Rep 16: 4038-4044, 2017.

21. Bonizzato A, Gaffo E, Te Kronnie G and Bortoluzzi S: CircRNAs in hematopoiesis and hematological malignancies. Blood Cancer J 6: e483, 2016.

(i) $\Theta$ This work is licensed under a Creative Common Attribution-NonCommercial-NoDerivatives 4.0 International (CC BY-NC-ND 4.0) License. 